\title{
3 Research Square \\ Low Expression of PDHX is Associated with Poor Prognosis and Immune Infiltration in Gastric Cancer
}

\section{Biao Sun (D274958615@qq.com )}

Yangzhou University

\section{Bao Li}

Yangzhou University

\section{Ziyang Long}

Dalian Medical University

\section{Cangyuan Zhang}

Dalian Medical University

\section{Qiannan Sun}

Dalian Medical University

Jun Ren

Northern Jiangsu People's Hospital

Daorong Wang

Northern Jiangsu People's Hospital

Research

Keywords: Gastric cancer, PDHX, Metabolic vulnerability, Warburg effect

Posted Date: September 27th, 2021

DOl: https://doi.org/10.21203/rs.3.rs-889509/v1

License: (c) (i) This work is licensed under a Creative Commons Attribution 4.0 International License.

Read Full License 


\section{Abstract}

Background: Pyruvate dehydrogenase protein X (PDHX) is a non-catalytic subunit of the pyruvate dehydrogenase (PDH) complex. It is located in the center of mitochondrial energy metabolism and is an essential component to maintain the biological activity of PDH complex. The purpose of this study was to explore its expression level in gastric cancer and its relationship with immune infiltration.

Methods: Through immunohistochemical analysis of 80 pairs of gastric cancer tissue samples and open database analys such as Kaplan-Meier Plotter, TIMER2.0,GEPIA,String and DAVID database.

Results: We found that the expression of PDHX in paracancerous tissues was significantly higher than that in gastric cancer tissues. Database analysis showed that the expression of PDHX was low in gastric cancer tissues, and the total survival time (OS) of relatively high expression in gastric cancer patients was higher. In N1 N3 and M stages, the P values of OS and PFS with high expression of PDHX were less than 0.05. It is suggested that the overexpression of PDHX may affect the prognosis of gastric cancer patients with lymph node and distant metastasis.

Conclusions: Therefore, we concluded that the expression of PDHX is suppressed in gastric cancer and has a longer overall survival time of 5 years in patients with relatively high expression, and that the increased expression of PDHX may improve the prognosis of patients with lymph node and distant metastasis of gastric cancer.

\section{Introduction}

Metabolic remodeling plays an important role in tumorigenesis and development. Its characteristics include glycolysis, glutamine metabolism, lipid metabolism, amino acid metabolism, mitochondrial respiration, nucleic acid biosynthesis, pentose phosphate pathway, autophagy-lysosome degradation pathway and other biosynthesis and bioenergy metabolism pathways $(1,2)$. The metabolites produced by these processes provide the necessary substances and energy for large-scale biosynthesis of tumor cells so that they can meet the needs of rapid proliferation, survival, dryness and metastasis of tumor cells(3). Because cancer is a disease mainly caused by heredity and gene mutations, these mutations include gene copy number aberrations such as gene amplification and deletion, as well as somatic mutations of metabolism-related genes, and metabolic remodeling is often caused by gene aberrations $(1-3)$. Therefore, in order to meet the huge energy and substrate needs of tumor cells, cell metabolism is often reprogrammed; however, this metabolic reprogramming often becomes some new weakness of the tumor, thus providing new opportunities for cancer treatment(1-3). So, the identification of essential genes for metabolism of tumor cells will contribute to the development of new cancer treatments for specific metabolic susceptibility.

Gastric cancer (GC) is the fifth leading cause of cancer-related death in the world, with high morbidity and mortality $(4,5)$. Based on the prominent recurrence rate of gastric cancer and the extremely low 5-year survival rate (late-stage $<10 \%$ ), understanding the progress of gastric cancer and the genetic factors 
involved in the late stage of the disease at the molecular level may help to discover new biomarkers and highlight the potential approach of targeted therapy. In most patients, gastric cancer is found to be in an advanced state and may have metastatic, which is one of the main reasons for its relatively poor prognosis. Therefore, the identification of new, reliable and potential molecular biomarkers is very important for the diagnosis or prognosis of patients with gastric cancer. In general, compared with normally differentiated cells, most cancer cells or undifferentiated cells (such as stem cells) mainly rely on aerobic glycolysis rather than mitochondrial oxidative phosphorylation for glucose metabolism, thus providing energy for cells $(6,7)$. This phenomenon was first described by Otto Weinberg in $1924(8)$, so it is called the "Warburg effect" (9). The Warburg effect observed that most cancer cells produce energy mainly through high-rate glycolysis and lactic acid fermentation in the cytoplasm, rather than through relatively little glycolysis and pyruvate oxidation in mitochondria as most normal cells do(10-12). Therefore, for tumors that often exist in the acidic microenvironment (TME), especially solid tumors, normal cells and tumor cells are usually affected by acidic microenvironment $(10,12,13)$. Pyruvate dehydrogenase protein $\mathrm{X}(\mathrm{PDHX})$ is a non-catalytic subunit of pyruvate dehydrogenase complex(PDHc). It is located in mitochondrial matrix and is the center of mitochondrial energy metabolism $(14,15)$. PDHc catalyzes the irreversible oxidation of pyruvate to acetyl coenzyme $A$, which is a rate-limiting step for the oxidative removal of glucose and pyruvate and other 3 carbon metabolites (alanine and lactic acid) in equilibrium with pyruvate under aerobic conditions(16). In addition to the non-catalytic subunit PDHX, the PDHc contains three catalytic subunits (pyruvate dehydrogenase E1, dihydrothiamide transacetylase E2 and dihydrothiamide dehydrogenase E3) and two regulatory subunits (E1 kinase and E1 phosphatase). It is well known that PDHX is a necessary component for binding E3 dimer to E2 core, and this specific binding is essential for functional PDH complexes. It plays an important role in cellular energy metabolism. It is reported that PDHX, as a ubiquitin E3 ligase, can convert pyruvate to acetyl-CoA and regulate glucose metabolism in colon cancer $(15,17)$. Previous studies have shown that inhibition of $\mathrm{PDHX}$ expression can promote the proliferation and invasion of breast cancer cells(18). In non-tumor diseases, PDHX has also been reported, for example, as an important component of PDHc activity, PDHX mutation plays an important role in congenital lactic acidosis(19). There is some correlation between lupus erythematosus and the expression of inflammatory factor CD44 in the pathogenesis of lupus erythematosus(20). Therefore, we can find that PDHX seems to play an important role in the occurrence, development of tumors, metabolic and immune diseases. Based on previous studies, we believe that PDHX may also play some role in gastric cancer and may become a potential molecular biomarker for the diagnosis, treatment or prognosis of gastric cancer. Therefore, the purpose of this study is to explore a new potential biomarker of gastric cancer.

\section{Material And Methods}

\section{Material source}

PDHX antibody was purchased from Wuhan Sanying Biotechnology Co., Ltd. We collected the clinical data and tissue sample of 80 patients with gastric cancer (Gastric Cancer, GC) who underwent surgical 
resection and pathologically confirmed in Northern Jiangsu People's Hospital from 2020 to 2021. This study has been approved by the Ethics Committee and the informed consent of the patient. All patients did not receive any treatment before an operation, and the tissue samples included gastric cancer tissue and corresponding paracancerous tissue. There were 68 males and 12 females, aged from 34 to 83 years old, with a median age of 67.5 years.

\section{Construction of tissue microarray}

We selected tissue samples from 80 patients with gastric cancer, each containing 2 sites (gastric cancer tissue and corresponding paracancerous tissue), a total of 160 sites, in order to make the tissue microarray we need. The tissue chip microarray was fabricated by Wuhan Seville Biotechnology Co., Ltd., and provided the patient sequence corresponding to each chip site. At the same time, we collected and recorded the clinicopathological data of 80 patients with gastric cancer, including sex, age, tumor location, clinicopathological grade, tumor stage, nerve invasion, vascular invasion and lymph node metastasis.

\section{Immunohistochemical (IHC) was used to detect the expression of PDHX}

Gastric cancer tissue microarray sections were baked in oven at $60^{\circ} \mathrm{C}$, dewaxed with xylene, rehydrated by gradient alcohol and pretreated with $3 \% \mathrm{H} 2 \mathrm{O} 2$, and then antigen epitopes were repaired with sodium citrate.

The slides were incubated overnight with rabbit anti-human PDHX (dilution ratio 1: 100; Wuhan Sanying Biotechnology Co., Ltd.) at $4^{\circ} \mathrm{C}$. The first antibody was discarded the next day, and the second antibody was incubated for 30 minutes at room temperature, and then horseradish peroxidase (HRP) was added to incubate $30 \mathrm{~min}$ at room temperature. Finally, the slices were visualized with freshly prepared DAB working solution (3meme 3-amino-diaminobenzidine, New cell $\square$ Molecular Biotech CO.,Ltd). Then the slides were re-dyed with hematoxylin, dehydrated with alcohol and xylene, and finally sealed with cover slides. Immune reaction using NDP.view2 detection system (Bimatsu Photonics Trading (China) Co., Ltd.) showed that the nucleus was re-stained with hematoxylin.

\section{Result Determination}

The staining area percentage and staining intensity of PDHX were graded independently by two senior pathologists and the final staining score was calculated. After evaluating the staining intensity of positive cells, the final results were obtained. This value is calculated based on the average percentage of PDHX positive tumor cells in the five regions after 200 -fold magnification. PDHX positive cells can be seen in different degrees of cytoplasmic staining in the tissue. It was scored according to the staining intensity $(0=$ negative; $1=$ light yellow; $2=\tan ; 3=$ brown) and the percentage of positive cells $(0$ : negative; $1:<=$ $10 \%, 2: 11 \%-50 \%, 3: 51 \%-75 \%, 4: 76-100 \%)$. The final score of each sample (0 12) is obtained by multiplying the staining intensity by the percentage of positive cells. The product of staining intensity and 
the percentage of positive cells greater than 3 is defined as positive, otherwise, it is negative. The median score was used as the cutoff value of high or low expression (PDHX median score $=6$ ). The tissue sample score greater than 6 is regarded as "high expression", and the score less than or equal to 6 is defined as "low expression".

\section{Kaplan-Meier Plotter Database Analysis}

The Kaplan Meier plotter is capable to assess the effect of 54k genes (mRNA, miRNA, protein) on survival in 21 cancer types including breast $(n=7,830)$, ovarian $(n=2,190)$, lung $(n=3,452)$, and gastric $(n=1,440)$ cancer. Sources for the databases include GEO, EGA, and TCGA. Primary purpose of the tool is a metaanalysis based discovery and validation of survival biomarkers. Here, we used Kaplan-Meier Plotter(Kaplan-Meier plotter (Gastric) (kmplot.com))to analyze the relationship between the expression of PDHX in gastric cancer and survival (21). The hazard ratio (HR) and logarithmic rank $\mathrm{P}$ of $95 \%$ confidence interval are calculated.

\section{TIMER database analysis}

The immune-gene module of TIMER2 database was used to explore the relationship between PDHX expression and immune infiltration of all tumors in TCGA database. CD8 + T cells, immune cells and tumor-associated fibroblasts were selected. TIMER, CiberSort, CiberSort-ABS, QuanTISEQ, XCell, MCPCOUNTER and EPIC algorithms were used to evaluate immune infiltration. The $P$ value and partial correlation (COR) value were obtained by purity-adjusted Spearman's rank correlation test. The data is presented in the form of heat maps and scatter maps.

\section{PDHX-related gene enrichment analysis}

First, log in to STRING (STRING: functional protein association networks (string-db.org)) database and query using a single protein name ("PDHX") and species ("Homo sapiens") information. We then set the following main parameters: the minimum required interaction score ("Low confidence ( 0.150 "), the meaning of the network edge ("evidence"), the maximum number of interaction objects to display ("no more than 50 interactors"), and the active interaction source ("experiments"). Finally, the available PDHX binding proteins were obtained.

Then, using the "Similar Gene Detection" module of GEPIA2, the first 100 target genes related to PDHX were obtained based on the data set of all TCGA tumors and normal tissues. We also used the "correlation analysis" module of GEPIA2 to analyze the Pearson correlation between PDHX and the selected genes. The scatter plot uses log2TPM, to give the $P$ value and the correlation coefficient $(R)$. In addition, we also use the "Gene_Corr" module of TIMER2 to provide the heat map data of the selected genes, including the partial correlation values (COR) and $\mathrm{P}$ values in the purity-adjusted Spearman rank correlation test.

Finally, we use Jvenn, an interactive Venn diagram viewer(22), to perform intersection analysis to compare genes that bind and interact with PDHX. 


\section{Statistical analysis}

All data are calculated using the SPSS26.0 statistical software (SPSS Inc.), Chicago, Illinois. For analysis. All data were expressed as mean \pm standard deviation and were statistically analyzed by student $t$ test. Kaplan-Meier method was used for survival analysis, and logarithmic rank test was used for survival difference. Pearson chi-square test and Continuity Correction test were used to evaluate and compare the clinicopathological features. $\mathrm{P}<0.05$ is considered to be statistically significant.

\section{Result}

\section{Inhibition of PDHX expression is associated with the progression of gastric cancer}

In order to study the expression of PDHX in gastric cancer, we first used immunohistochemical staining method to detect the protein expression of stage $\triangle-\rrbracket$ and stage $\rrbracket-\bigotimes$ PDHX in gastric cancer tissues and corresponding paracancerous tissues. It can be found that PDHX is expressed in the cytoplasm of cells. And the positive expression rate of PDHX in paracancerous tissues was $88.8 \%$ (71 to 80 ), which was significantly higher than that in gastric cancer (73.8\%) (Table 1 and Fig. 1B).

Table 1

Expression of PDHX in Normal and Cancer tissues.

\begin{tabular}{|c|c|c|c|c|c|}
\hline \multirow[t]{2}{*}{ Tissue Sample } & & PDHX & Expression & & \\
\hline & $\mathrm{n}$ & Active & Negative & $x^{2}$ & $\mathbf{P}$ \\
\hline Normal Tissue & 80 & $71(88.75)$ & $9(11.25)$ & 5.908 & 0.015 \\
\hline Cancer Tissue & 80 & $59(73.75)$ & $21(26.25)$ & & \\
\hline
\end{tabular}

In addition, we also studied the potential correlation between the expression of PDHX and the clinicopathological features of patients with gastric cancer. As shown in Table 2, there was no significant correlation between the expression of PDHX and gender, tumor diameter, depth of invasion, lymph node invasion and nerve invasion. However, it was negatively correlated with age, differentiation degree, TNM stage, lymph node metastasis and vascular invasion, and the inhibition of PDHX expression was more

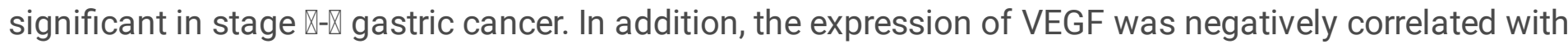
age $(P=0.002)$, differentiation $(P=0.001)$, TNM stage $(P=0.032)$, lymph node metastasis $(N$ stage $P=$ $0.013)$ and vascular invasion $(P=0.026)$, and the inhibition of VEGF expression was more significant in stage $\mathbb{X}-\mathbb{Q}$ gastric cancer. 
Table 2

Relationship between PDHX protein expression and clinicopathologic features of patients [n (\%)]

\begin{tabular}{|c|c|c|c|c|}
\hline Parameters & $\operatorname{High}(n=26)$ & $\operatorname{Low}(n=54)$ & $x^{2}$ & $\mathbf{P}$ \\
\hline SEX & & & 1.144 & 0.285 \\
\hline Female & $20(76.92)$ & 48(88.89) & & \\
\hline Male & $6(23.08)$ & $6(11.11)$ & & \\
\hline Age(years) & & & 9.95 & 0.002 \\
\hline$>=65$ & $24(92.31)$ & $31(57.41)$ & & \\
\hline$<65$ & $2(7.69)$ & 23(42.59) & & \\
\hline Tumor diameter & & & 1.899 & 0.168 \\
\hline$>=5$ & 17(65.39) & 43(79.63) & & \\
\hline$<5$ & $9(34.61)$ & 11(20.37) & & \\
\hline Lymph node invasi & & & 2.65 & 0.104 \\
\hline $\mathrm{N} 0+\mathrm{N} 1$ & $12(46.15)$ & 15(27.78) & & \\
\hline $\mathrm{N} 2+\mathrm{N} 3$ & 14(53.85) & $39(72.22)$ & & \\
\hline Infiltration depth & & & 0 & 1 \\
\hline $\mathrm{T} 1+\mathrm{T} 2$ & $3(11.54)$ & $5(9.26)$ & & \\
\hline $\mathrm{T} 3+\mathrm{T} 4$ & $23(88.46)$ & $49(90.74)$ & & \\
\hline Differentiation & & & 10.43 & 0.001 \\
\hline $\mathrm{G} 1+\mathrm{G} 2$ & $14(53.85)$ & $10(18.52)$ & & \\
\hline G3 & $12(46.15)$ & $44(81.48)$ & & \\
\hline TNM & & & 4.603 & 0.032 \\
\hline 『/ष & $10(38.46)$ & $9(16.67)$ & & \\
\hline 『/ष & 16(61.54) & 45(83.33) & & \\
\hline Nerve invasion & & & 0.09 & 0.764 \\
\hline Yes & 14(53.85) & $31(57.41)$ & & \\
\hline No & $12(46.15)$ & 23(42.59) & & \\
\hline Vascular invasion & & & 4.953 & 0.026 \\
\hline Yes & $10(38.46)$ & $35(64.81)$ & & \\
\hline
\end{tabular}




\begin{tabular}{|c|c|c|c|c|}
\hline Parameters & $\operatorname{High}(n=26)$ & $\operatorname{Low}(n=54)$ & $x^{2}$ & $\mathbf{P}$ \\
\hline No & $16(61.54)$ & 19(35.19) & & \\
\hline \multicolumn{3}{|c|}{ Lymph node metastasis } & 6.154 & 0.013 \\
\hline Yes & $15(57.69)$ & 45(83.33) & & \\
\hline No & $11(42.31)$ & $9(16.67)$ & & \\
\hline
\end{tabular}

\section{Expression and Prognostic Analysis of PDHX in gastric Cancer}

The expression and prognosis of PDHX in gastric cancer can be obtained from the website (www.kmplot.com). According to the Affymetrix ID:203067_at (PDHX) analysis of the data set. It can be seen that the expression of PDHX in gastric cancer tissues is lower than that in paracancerous tissues (Fig. 2A, B). The high expression of PDHX-mRNA was correlated with better OS and FPS in gastric cancer (Fig. 2C, D).

\section{Effect of high expression of PDHX on prognosis of patients with gastric cancer with lymph node and distant metastasis}

In order to better understand the correlation and potential mechanism of PDHX expression in gastric malignant tumors, we studied the relationship between PDHX expression and clinical characteristics of patients with gastric cancer in Kaplan-Meier Plotter database. In male and female patients, the overexpression of PDHX was related to the deterioration of OS and PFS and the classification of Lauren $(\mathrm{P}<0.05)$. The high expression of PDHX-mRNA was associated with the deterioration of OS and PFS in patients with stage 3-4 gastric cancer, but not with stage 1-2 gastric cancer (Table 3 ). In the TNM staging of the tumor, $\mathrm{T}$ refers to the depth of tumor invasion; N refers to lymph node metastasis; N0 indicates no regional lymph node metastasis and N1-N3 indicates regional lymph node metastasis. $\mathrm{M}$ means distant metastasis. In N1 N3 and M classification, the P values of OS and PFS highly expressed by PDHX were less than 0.05 (Table 3). It is suggested that the expression level of PDHX may affect the prognosis of patients with gastric cancer with lymph node and distant metastasis. 
Table 3

Correlation of PDHXmRNA expression and clinical prognosis in gastric cancer with different clinicopathological factors by Kaplan-Meier plotter.

\section{Clinicopathological} characteristcs

\begin{tabular}{|c|c|c|c|c|c|c|}
\hline & N & Hazard ratio & P-value & $\mathrm{N}$ & Hazard ratio & P-value \\
\hline \multicolumn{7}{|l|}{ SEX } \\
\hline Female & 244 & $\begin{array}{l}0.49(0.34- \\
0.71)\end{array}$ & 0.00013 & 244 & $\begin{array}{l}0.49(0.33- \\
0.73)\end{array}$ & $\begin{array}{l}3.00 \mathrm{E}- \\
04\end{array}$ \\
\hline Male & 566 & $\begin{array}{l}0.61(0.49- \\
0.76)\end{array}$ & $\begin{array}{l}9.60 \mathrm{E}- \\
06\end{array}$ & 566 & $\begin{array}{l}0.6(0.46- \\
0.78)\end{array}$ & 0.00012 \\
\hline \multicolumn{7}{|l|}{ STAGE } \\
\hline 1 & 69 & $\begin{array}{l}0.42(0.14- \\
1.31)\end{array}$ & 0.13 & 69 & $\begin{array}{l}0.45(0.13- \\
1.6)\end{array}$ & 0.21 \\
\hline 2 & 145 & $\begin{array}{l}0.69(0.36- \\
1.33)\end{array}$ & 0.26 & 145 & $\begin{array}{l}1.75(0.89- \\
3.42)\end{array}$ & 0.098 \\
\hline 3 & 319 & $\begin{array}{l}0.61(0.45- \\
0.81)\end{array}$ & 0.00062 & 319 & $\begin{array}{l}0.56(0.34- \\
0.91)\end{array}$ & 0.017 \\
\hline 4 & 152 & $\begin{array}{l}0.59(0.4- \\
0.87)\end{array}$ & 0.007 & 152 & $\begin{array}{l}0.58(0.38- \\
0.89)\end{array}$ & 0.01 \\
\hline \multicolumn{7}{|l|}{ STAGE T } \\
\hline 2 & 253 & $\begin{array}{l}0.69(0.44- \\
1.07)\end{array}$ & 0.099 & 253 & $\begin{array}{l}0.69(0.45- \\
1.06)\end{array}$ & 0.091 \\
\hline 3 & 208 & $\begin{array}{l}0.67(0.46- \\
0.960\end{array}$ & 0.028 & 208 & $\begin{array}{l}0.77(0.54- \\
1.1)\end{array}$ & 0.15 \\
\hline 4 & 39 & $\begin{array}{l}0.47(0.18- \\
1.27)\end{array}$ & 0.13 & 39 & $\begin{array}{l}0.34(0.14- \\
0.82)\end{array}$ & 0.012 \\
\hline \multicolumn{7}{|l|}{ STAGE N } \\
\hline 0 & 76 & $\begin{array}{l}0.63(0.23- \\
1.72)\end{array}$ & 0.36 & 76 & $\begin{array}{l}0.49(0.19- \\
1.26)\end{array}$ & 0.13 \\
\hline 1 & 232 & $\begin{array}{l}0.54(0.34- \\
0.85)\end{array}$ & 0.007 & 232 & $\begin{array}{l}0.65(0.42- \\
0.99)\end{array}$ & 0.046 \\
\hline 2 & 129 & $\begin{array}{l}0.48(0.3- \\
0.76)\end{array}$ & 0.0014 & 129 & $\begin{array}{l}0.48(0.31- \\
0.75)\end{array}$ & 0.00085 \\
\hline 3 & 76 & $\begin{array}{l}0.33(0.16- \\
0.68)\end{array}$ & 0.0016 & 76 & $\begin{array}{l}0.36(0.18- \\
0.72)\end{array}$ & 0.0028 \\
\hline $1+2+3$ & 437 & $\begin{array}{l}0.57(0.43- \\
0.77)\end{array}$ & 0.00015 & 437 & $\begin{array}{l}0.66(0.5- \\
0.87)\end{array}$ & 0.0027 \\
\hline
\end{tabular}

Overall survival $(n=$ 881)

\section{Progression-free survival $(n=645)$ \\ $(n=645)$}




\begin{tabular}{|c|c|c|c|c|c|c|}
\hline \multirow{2}{*}{$\begin{array}{l}\text { Clinicopathological } \\
\text { characteristcs }\end{array}$} & & \multicolumn{3}{|c|}{$\begin{array}{l}\text { Overall survival }(n= \\
\text { 881) }\end{array}$} & \multicolumn{2}{|c|}{$\begin{array}{l}\text { Progression-free surviva } \\
(\mathrm{n}=645)\end{array}$} \\
\hline & & & & & & \\
\hline 0 & 459 & $\begin{array}{l}0.64(0.45- \\
0.91)\end{array}$ & 0.013 & 459 & $\begin{array}{l}0.64(0.45- \\
0.9)\end{array}$ & 0.0099 \\
\hline 1 & 58 & $\begin{array}{l}0.27(0.13- \\
0.55)\end{array}$ & 0.00013 & 58 & $\begin{array}{l}0.5(0.26- \\
0.95)\end{array}$ & 0.031 \\
\hline \multicolumn{7}{|c|}{ LAUREN CLASSIFICATION } \\
\hline Intestinal & 336 & $\begin{array}{l}0.47(0.33- \\
0.65)\end{array}$ & $\begin{array}{l}3.40 \mathrm{E}- \\
06\end{array}$ & 336 & $\begin{array}{l}0.62(0.43- \\
0.89)\end{array}$ & 0.0095 \\
\hline Diffuse & 248 & $\begin{array}{l}0.59(0.39- \\
0.91)\end{array}$ & 0.015 & 248 & $\begin{array}{l}0.61(0.4- \\
0.91)\end{array}$ & 0.015 \\
\hline \multicolumn{7}{|l|}{ DIFFERENTIATION } \\
\hline Poor & 166 & $\begin{array}{l}0.82(0.53- \\
1.26)\end{array}$ & 0.37 & 166 & $\begin{array}{l}0.74(0.47- \\
1.19\end{array}$ & 0.22 \\
\hline Moderate & 67 & $\begin{array}{l}0.57(0.28- \\
1.17)\end{array}$ & 0.12 & 67 & $\begin{array}{l}0.47(0.24- \\
0.92)\end{array}$ & 0.024 \\
\hline
\end{tabular}

\section{Analysis of tumor immune infiltration}

As an important part of tumor microenvironment, tumor infiltrating immune cells are closely related to the occurrence, development or metastasis of tumor $(23,24)$. It is reported that tumor-associated fibroblasts in tumor microenvironment stroma are involved in regulating the function of various tumor infiltrating immune cells $(25,26)$. Here, we use TIMER, X-cell, MCPCOUNTER and TIDE algorithms to study the potential relationship between different levels of immune cell infiltration and PDHX gene expression in different types of TCGA tumors. In addition, we observed that the expression of PDHX-mRNA was positively correlated with the infiltration of tumor-related fibroblasts of HNSC and HNSC-HPV -, but negatively correlated with BRCA, STAD (Fig. 3).

\section{Enrichment analysis of PDHX related genes}

In order to further study the molecular mechanism of PDHX gene in tumorigenesis, we tried to screen out targeted PDHX binding proteins and PDHX expression related genes for a series of pathway enrichment analysis. Based on the STRING tool, we obtained a total of 50 PDHX-binding proteins supported by experimental evidence and constructed the PPI network of these proteins (Fig. 4A). Using GEPIA2 tool combined with all tumor expression data of TCGA, we obtained the first 100 genes related to PDHX expression. The expression level of PDHX was positively correlated with that of CS, DLD, DLAT, OGDH, PDHA1 and PDHB (Fig. 4B). The corresponding heat map data also showed that in most detailed cancer 
types, PDHX was positively correlated with the above six genes (Fig. 4C). The cross-analysis of the above two groups shows that there are six common members, namely, CS, DLD, DLAT, OGDH, PDHA1, and PDHB (Fig. 4D). We combine two data sets for KEGG enrichment analysis. As shown in Fig. 4e, PDHX is mainly involved in Citrate cycle (TCA cycle) and 2-oxocarboxylic acid metabolism pathway, which affects the pathogenesis or progression of tumor.

\section{Discussion}

PDHX pyruvate dehydrogenase protein X (PDHX), as a non-catalytic subunit ofPDHc, is located in the mitochondrial matrix and at the center of mitochondrial energy metabolism. Its role in tumorigenesis and development seems to be very complex, because it has both carcinogenic and tumor inhibitory effects in cancer. For example, as a direct target gene of mir-26a and mir-27b, PDHX down-regulates the growth and metastatic potential of colorectal and breast cancer cells $(15,18)$. It is also reported that PDHX, as an important metabolic gene in esophageal squamous cell carcinoma, is necessary to maintain the activity of PDHc and produce ATP. Its knockdown inhibits the proliferation and tumor growth of esophageal squamous cell carcinoma stem cell (CSCs)(27). In non-tumor diseases, PDHX has also been reported, for example, as an important component of PDHc activity, PDHX mutation plays an important role in congenital lactic acidosis(19). There is some synergism between lupus erythematosus and the expression of inflammatory factor CD44 in the pathogenesis of lupus erythematosus(20). From these studies, we can find that PDHX seems to play some important roles in the occurrence and development of tumors and metabolic and immune diseases. Based on the study of PDHX in tumor and non-tumor metabolic and immune diseases, we believe that PDHX may play some role in immune evasion of gastric cancer, so as mentioned above, we used Timer2.0 database to study the potential relationship between different levels of immune cell infiltration and PDHX gene expression in different types of TCGA tumors. In addition, as the hub of glycolysis and tricarboxylic acid cycle, the low expression of PDHX in cancer cells also inhibits the activity of PDHc. As a result, pyruvate accumulates upstream, reducing downstream acetyl CoA production and diverting it to lactic acid production, providing continuous glycolysis flux or feeding cells for biomolecule synthesis (Fig. 5). The loss of PDH function also leads to a decrease in aerobic respiration, which indicates that cancer cells become more dependent on the aerobic glycolysis pathway. This is consistent with previous reports, which confirmed that impaired PDHc function can trigger malignant metabolic phenotypes of tumors(28). This change, as well as the increase in lactic acid production, is a unique feature of the Weinberg effect. A series of hypotheses put forward so far in this field suggest that this energy metabolic remodeling is beneficial to the growth of tumor volume(29).

Energy metabolism remodeling is the characteristic of energy metabolism of tumor cells and their neighboring cells in tumor microenvironment (TME). The latter includes stromal fibroblasts and adipocytes, which differentiate into cancer-associated fibroblasts (CAF) and cancer-associated adipocytes (CAA) as well as immune infiltrating cells. They cooperate with stromal cells to provide a favorable living environment for tumor growth. Cancer-associated fibroblasts (CAF) are located near the tumor and account for a large part of the tumor mass. It is reported that CAF accounts for $80 \%$ of tumor masses in breast and pancreatic cancer(30). CAF has its genetic characteristics and has the ability to 
influence tumorigenesis, invasion and progression by releasing cytokines, chemokines and angiogenic factors(31). It should also be noted that very similar to tumor cells, CAF undergoes metabolic remodeling and changes to glycolysis phenotype, which enables them to produce high-energy metabolites such as lactic acid, pyruvate and ketone bodies, which provides fuel for neighboring tumor cells(32). Energy metabolism reprogramming is related to TME acidification, and TME acidification is also an important feature of tumor progression $(33,34)$. And the establishment of tumor acidic microenvironment (TME) reduces the efficiency or loss of function of most anti-tumor immune cells(35). In addition to inhibiting glycolysis of PDHX, tumors, it may also be disturbed by any intermediate step in glucose metabolism. For example, it is known that inhibiting hexokinase (36)or any other enzyme that catalyzes the subsequent reaction of glucose metabolism can enhance the anticancer effect $(37,38)$. Removing the regulation of tumor glycolysis by targeting glycolytic enzymes other than PDHX may also have additional ideal results. This is mainly due to the "feedback" inhibition mechanism of glucose metabolism. Specifically, the metabolites of the intermediate steps of glycolysis accumulate due to the inhibition of specific enzymes, and finally block or slow down the rate of glucose catabolism in the way of negative feedback. Therefore, the destruction of tumor glycolysis seems to reduce the oxidation and utilization of glucose.

Although aerobic glycolysis may not produce as much ATP as mitochondria, it has the advantages of higher efficiency, use of unconventional nutrients and non-dependence on oxygen. Moreover, malignant tumor cells for the purpose of self-proliferation, its gene mutation will be more conducive to the effective acquisition of macromolecular substances for self-proliferation, rather than energy production. The rapid proliferation of malignant tumor cells also needs a lot of energy, but its degree of differentiation is low, and it may lack the key enzymes necessary for the process of oxidative phosphorylation, so we have to choose glycolysis to supply energy. Compared with the oxidative phosphorylation of glucose, glycolysis can be completed in the cytoplasm without entering the mitochondria for metabolism, reducing the key enzymes needed in the metabolic process. At the same time, because of its simple metabolic process, it can provide energy for rapidly proliferating cells more quickly(39). The relatively hypoxic environment of tumor tissue makes glycolysis an effective choice, and the lactic acid it produces can provide an acidic microenvironment for tumor cells. This acidic microenvironment can induce immune escape, promote invasion and metastasis of tumor cells, while at the same time promote apoptosis of normal cells, turn off DNA repair mechanism, and inhibit apoptosis of tumor cells(40). The decrease of oxidative phosphorylation in tumor cells leads to a decrease in the production of reactive oxygen species (ROS) to some extent, thus reducing the killing effect on tumor cells(41-43). Malignant tumor cells choose glycolysis pathway to provide themselves with raw materials and energy for proliferation, and produce acidic microenvironment and reduce reactive oxygen species (ROS) to inhibit apoptosis, which is not only an important cause of tumorigenesis, to a certain extent, it is more likely to be the result of tumor cell evolution, which will be more conducive to tumor cell invasion, proliferation and metastasis.

Gastric cancer ,as fifth-largest cancer in the world and third-largest cancer in China, has the characteristics of high morbidity and high mortality $(4,5)$. With the invasive invasion or lymphatic metastasis of gastric cancer, although traditional therapy, such as surgery, radiotherapy, chemotherapy or traditional Chinese medicine, is used to enhance the therapeutic effect, the therapeutic effect of gastric 
cancer is still not satisfactory(44). In the face of such a severe situation, it is urgent to develop new treatment methods to improve the diagnosis rate and judge the prognosis of gastric cancer. In order to make significant improvements in the treatment of gastric cancer, we must be willing to study new and unconventional treatment methods. There is growing evidence that abnormal cell metabolism is a basic component of tumor composition, which increases the possibility that treatments developed for this feature will be significantly effective in the future(45). In fact, it has been added to the list of cancer markers by Hanahan and Weinberg, so its importance in the pathogenesis of cancer is evident(46).At present, some limited evidence shows that metabolically active drugs have therapeutic effects on cancer. For example, clinical studies have reported that diabetics who use metformin to control hyperglycemia have a lower risk of breast cancer $(47,48)$

In short, the energy metabolism of tumor cells is reconstructed, the expression of enzymes related to glycolysis decreases or increases, and some genes are mutated, which may become targets of anti-tumor therapy. Hope and challenges coexist, tumor is essentially a group of cells with metabolic changes, and the treatment of tumor cell metabolism may become a new milestone in anti-tumor therapy.

\section{Authors information}

Clinical Medical College, Yangzhou University, Yangzhou 225001, China.

Biao Sun, Li Bao ,Daorong Wang

Dalian Medical University, Dalian116044, China.

Ziyang Long, Cangyuan Zhang

Northern Jiangsu People's Hospital, Yangzhou 225001, China.

Qiannan Sun, Jun Ren, Daorong Wang

General Surgery Institute of Yangzhou, Yangzhou University,Yangzhou 225001,China.

Qiannan Sun, Jun Ren,Daorong Wang

\section{Availability of data and materials}

The datasets used and/or analysed during the current study are available from the corresponding author on reasonable request.

\section{Abbreviations}

PDHX

Pyruvate dehydrogenase protein $\mathrm{X}$

$\mathrm{PDHc}$ 
Pyruvate dehydrogenase complex

TME

Tumor microenvironment

$\mathrm{CAF}$

Cancer-associated fibroblasts

CAA

Cancer-associated adipocytes

ROS

Reactive oxygen species

\section{Declarations}

\section{Authors information}

Clinical Medical College, Yangzhou University, Yangzhou 225001, China.

Biao Sun, Li Bao ,Daorong Wang

Dalian Medical University, Dalian116044, China.

Ziyang Long, Cangyuan Zhang

Northern Jiangsu People's Hospital, Yangzhou 225001, China.

Qiannan Sun, Jun Ren, Daorong Wang

General Surgery Institute of Yangzhou, Yangzhou University,Yangzhou 225001,China.

Qiannan Sun, Jun Ren,Daorong Wang

\section{Availability of data and materials}

The datasets used and/or analysed during the current study are available from the corresponding author on reasonable request.

\section{Ethics declarations}

The study protocol was approved by the Medical Ethics Committee of Northern Jiangsu people's Hospital(Due to the prevalence of COVID-19, the work of the committee has been temporarily affected and the approval documents have not been obtained for the time being.

). In addition, the study has obtained the informed consent of all participants. 


\section{Consent for publication}

All participating patients agreed to publish their data.

\section{FUNDING}

This work was partially supported by the National Natural Science Foundation of China (81972269).

\section{Availability of data and materials}

The datasets used and/or analyzed during the present study are available from the corresponding author on reasonable request.

\section{Declaration of Competing Interest}

The authors declare that they have no competing interest.

\section{Acknowledgements}

Biao Sun contributed to the conception and drafting of the manuscript; Li Bao et al. helped with immunohistification experiments. Jun Ren and Qiannan Sun revised the manuscript and approved the final version. All authors have read and approved the final version for publication.

\section{References}

1. Ferlay J, et al., Cancer incidence and mortality worldwide: Sources, methods and major patterns in GLOBOCAN 2012. International Journal of Cancer, 2015. 136(5).

2. Sharma AK, Eils R, KÖnig R. Copy Number Alterations in Enzyme-Coding and Cancer-Causing Genes Reprogram Tumor Metabolism. Cancer Research, 2016. 76(14).

3. Phan LM, Yeung S-CJ, Lee M-H. Cancer metabolic reprogramming: importance, main features, and potentials for precise targeted anti-cancer therapies. Cancer Biology Medicine. 2014;11(01):1-19.

4. Rahib L, et al. Projecting Cancer Incidence and Deaths to 2030: The Unexpected Burden of Thyroid, Liver, and Pancreas Cancers in the United States. Can Res. 2014;74(14):2913-21.

5. Siegel RL, Miller KD, Jemal A, Cancer statistics, 2018. CA: A Cancer Journal for Clinicians, 2018. 68(1).

6. Heiden M, Cantley LC, Thompson CB. Understanding the Warburg Effect: The Metabolic Requirements of Cell Proliferation. Science. 2009;324(5930):1029-33.

7. Über den Stoffwechsel von Tumoren im Körper. Klinische Wochenschrift, 1926. 5(19): p. 829-832.

8. Warburg O. ber den Stoffwechsel der Carcinomzelle. Naturwissenschaften. 1924;12(50):1131-7.

9. Johnson LG, Newell RC. Temperature-compensated Oxygen Consumption of Subcellular Preparations from Vertebrate Ectotherm, Endotherm, and Tumor Tissues. Berlin Heidelberg: Springer; 1973. 
10. Corbet C, Feron 0. Tumour acidosis: from the passenger to the driver's seat. Nature Reviews Cancer, 2017.

11. Shanmugasundaram GK. Taming Tumor Glycolysis and Potential Implications for Immunotherapy. Frontiers in Oncology, 2017. 7.

12. Ping W, et al. Increased Lactate in Gastric Cancer Tumor-Infiltrating Lymphocytes Is Related to Impaired T Cell Function Due to miR-34a Deregulated Lactate Dehydrogenase A. Cell Physiol Biochem. 2018;49:828-36.

13. Anderson, et al., Obstacles Posed by the Tumor Microenvironment to T cell Activity: A Case for Synergistic Therapies. Cancer Cell, 2017.

14. Stacpoole PW. The pyruvate dehydrogenase complex as a therapeutic target for age-related diseases. Aging Cell. 2012;11(3):371-7.

15. Bing $\mathrm{C}$, et al. MicroRNA-26a regulates glucose metabolism by direct targeting PDHX in colorectal cancer cells. Bmc Cancer. 2014;14(1):443.

16. Margineantu $\mathrm{DH}$, et al. Heterogeneous distribution of pyruvate dehydrogenase in the matrix of mitochondria. Mitochondrion. 2002;1(4):327-38.

17. Park YH, Patel MS. Characterization of interactions of dihydrolipoamide dehydrogenase with its binding protein in the human pyruvate dehydrogenase complex. Biochemical Biophysical Research Communications. 2010;395(3):416-9.

18. Eastlack SC, et al. Suppression of PDHX by microRNA-27b deregulates cell metabolism and promotes growth in breast cancer. Molecular Cancer. 2018;17(1):100.

19. Ivanov IS, et al., Founder p.Arg 446* mutation in the PDHX gene explains over half of cases with congenital lactic acidosis in Roma children. Molecular Genetics and Metabolism, 2014. 113(1-2).

20. Lessard CJ, et al., Identification of a Systemic Lupus Erythematosus Susceptibility Locus at 11p13 between PDHX and CD44 in a Multiethnic Study. The American Journal of Human Genetics, 2011. 88(1).

21. Marcell SA, et al., Cross-validation of survival associated biomarkers in gastric cancer using transcriptomic data of 1,065 patients. Oncotarget, 2016. 7(31).

22. Philippe B, et al., jvenn: an interactive Venn diagram viewer. BMC bioinformatics, 2014. 15.

23. Fridman $W$, et al. Immune infiltration in human cancer: prognostic significance and disease control. current topics in microbiology immunology. 2011;344(1):1.

24. Steven A, Seliger B, The Role of Immune Escape and Immune Cell Infiltration in Breast Cancer. Breast Care, 2018.

25. Chen X, Song E. Turning foes to friends: targeting cancer-associated fibroblasts. Nature Reviews Drug Discovery, 2018. 18(2).

26. Kwa MQ, Herum KM, Brakebusch C. Cancer-associated fibroblasts: how do they contribute to metastasis? Clinical \& Experimental Metastasis; 2019. 
27. Jun I, et al., Identification of PDHX as a metabolic target for esophageal squamous cell carcinoma. Cancer science, 2021.

28. Mcfate T, et al. Pyruvate Dehydrogenase Complex Activity Controls Metabolic and Malignant Phenotype in Cancer Cells. J Biol Chem. 2008;283(33):22700-8.

29. Liberti MV, Locasale JW. The Warburg Effect: How Does it Benefit Cancer Cells? Trends in Biochemical Sciences, 2016. 41(3).

30. Alessandro A, et al., Cancer: An Oxidative Crosstalk between Solid Tumor Cells and Cancer Associated Fibroblasts. BioMed Research International,2016,(2016-8-9), 2016. 2016: p. 1-7.

31. Lea M, Neta E. The Dark Side of Fibroblasts: Cancer-Associated Fibroblasts as Mediators of Immunosuppression in the Tumor Microenvironment. Frontiers in Immunology; 2019.

32. Martinez-Outschoorn UE, Lisanti MP, Sotgia F. Catabolic cancer-associated fibroblasts transfer energy and biomass to anabolic cancer cells, fueling tumor growth. Seminars in Cancer Biology, 2014. 25.

33. Bianchini, et al. The acidic microenvironment as a possible niche of dormant tumor cells. Cellular: CMLS; 2017.

34. Peppicelli S, Bianchini F, Calorini L. Extracellular acidity, a "reappreciated" trait of tumor environment driving malignancy: perspectives in diagnosis and therapy. Cancer Metastasis Rev. 2014;33(23):823-32.

35. Kareva I, Hahnfeldt P. The Emerging \"Hallmarks\" of Metabolic Reprogramming and Immune Evasion: Distinct or Linked? Can Res. 2013;73(9):2737-42.

36. Mathupala SP, Ko YH, Pedersen PL. Hexokinase II: Cancer's double-edged sword acting as both facilitator and gatekeeper of malignancy when bound to mitochondria. Oncogene. 2006;25(34):4777-86.

37. Ganapathy-Kanniappan S, Kunjithapatham R, Geschwind JF. Glyceraldehyde-3-Phosphate Dehydrogenase: A Promising Target for Molecular Therapy in Hepatocellular Carcinoma. Oncotarget. 2012;3(9):940-53.

38. Roberto, et al., Glycolytic enzyme inhibitors in cancer treatment. Expert opinion on investigational drugs, 2008. 17(10): p. 1533-45.

39. Heiden MGV, Cantley LC, Thompson CB. Understanding the Warburg Effect: The Metabolic Requirements of Cell Proliferation. Science, 2009. 324(5930).

40. Gu Z-W, et al., MiR-1180 from bone marrow-derived mesenchymal stem cells induces glycolysis and chemoresistance in ovarian cancer cells by upregulating the Wnt signaling pathway. Zhejiang University Press, 2019. 20(3).

41. Edge L, Cancer Cell Metabolism: Warburg and Beyond. 2008.

42. B AJL,MT. and Q.C. A, The Warburg effect in tumor progression: Mitochondrial oxidative metabolism as an anti-metastasis mechanism - ScienceDirect. Cancer Lett. 2015;356(2):156-64. 
43. Vaupel P, Schmidberger $\mathrm{H}$, Mayer A. The Warburg effect: Essential part of metabolic reprogramming and central contributor to cancer progression. Int J Radiat Biol. 2019;95(7):1-25.

44. Liu X, et al. Traditional Chinese medicine integrated with chemotherapy for stage IV non-surgical gastric cancer: a retrospective clinical analysis. Journal of Integrative Medicine. 2017;15(6):469-75.

45. Kroemer G, Pouyssegur J. Tumor cell metabolism: cancer's Achilles' heel. Cancer Cell. 2008;13(6):472-82.

46. Weinberg H, Hallmarks of Cancer: The Next Generation. Cell, 2011.

47. Noto H, et al., Cancer Risk in Diabetic Patients Treated with Metformin: A Systematic Review and Meta-analysis. Plos One, 2012. 7.

48. Joungyoun K, et al., Metformin use reduced the risk of stomach cancer in diabetic patients in Korea: an analysis of Korean NHIS-HEALS database. Gastric cancer: official journal of the International Gastric Cancer Association and the Japanese Gastric Cancer Association, 2020. 23(6).

\section{Figures}
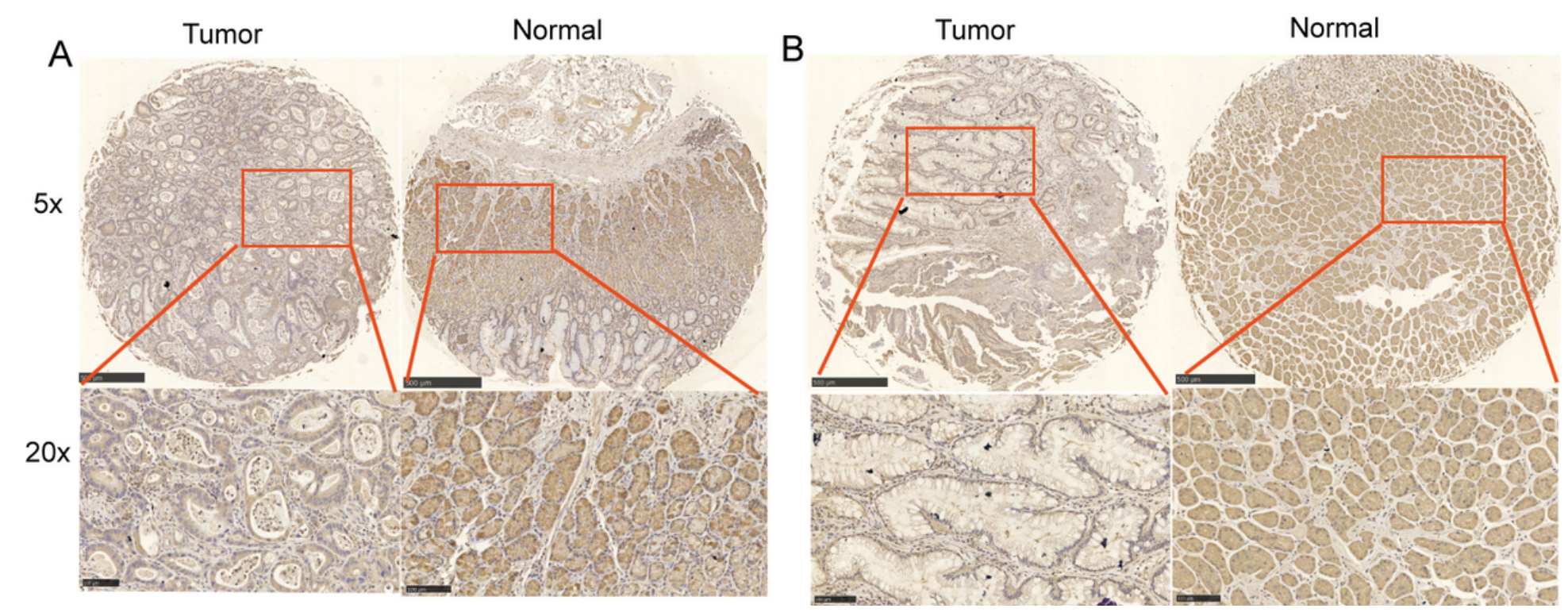

Figure 1

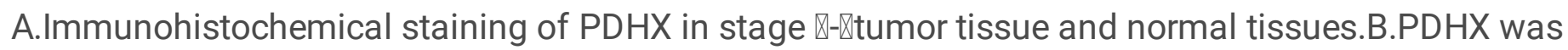
stained by immunohistochemistry in stage $\mathbb{X} \mathbb{X}$ tumor tissue and normal tissues. 
A

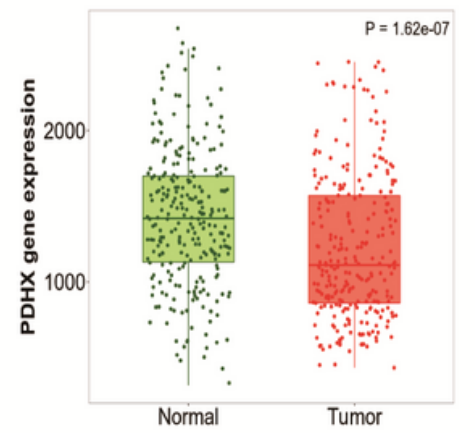

C

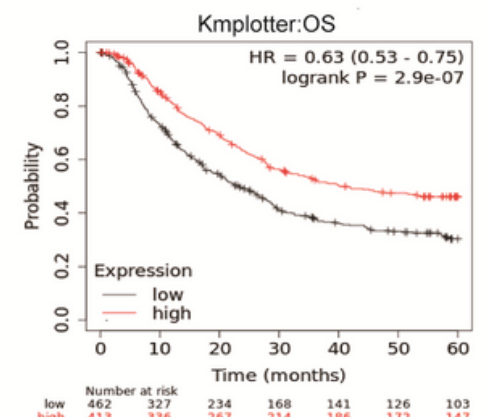

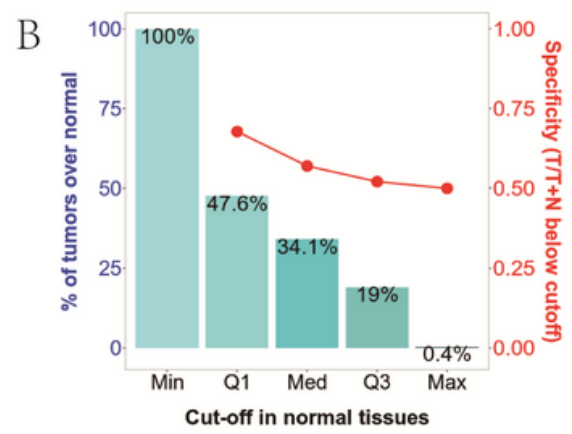

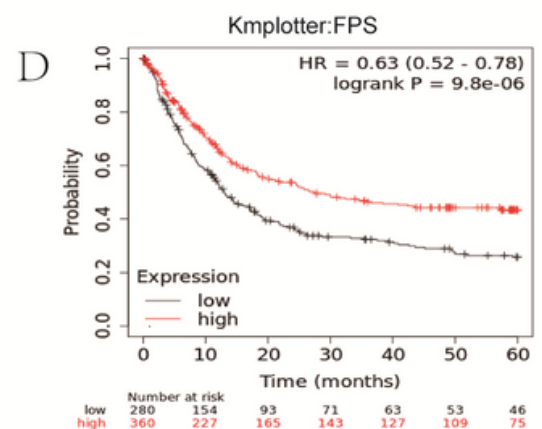

Figure 2

A. The differential expression of PDHX in gastric cancer and paracancerous tissues.B. The cutoff value of differential expression of PDHX in gastric cancer and paracancerous tissues.C. The 5-year overall survival rate of PDHX expression in patients with gastric cancer was demonstrated.D.The 5-year progression-free survival of PDHX expression in patients with gastric cancer was demonstrated. 

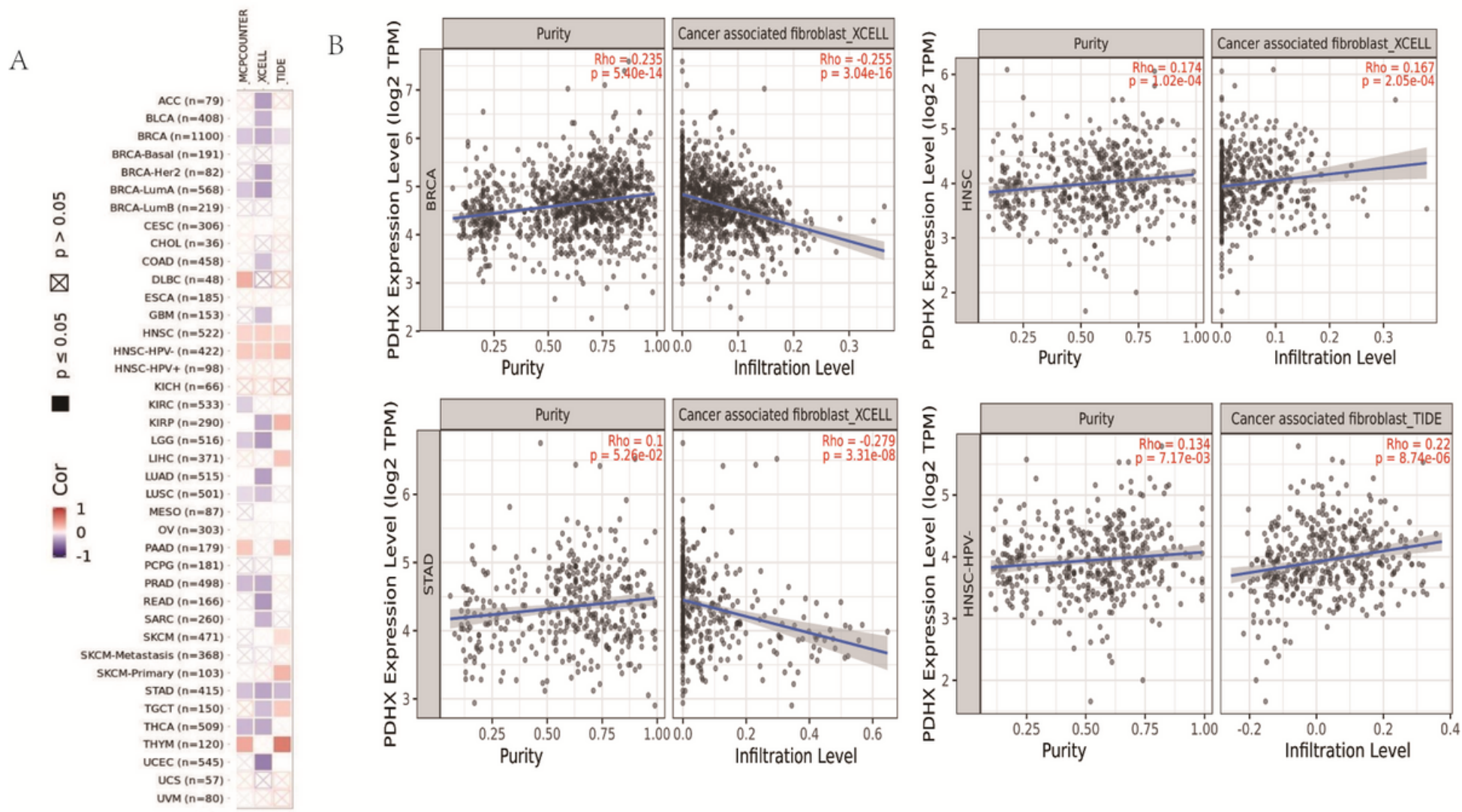

Figure 3

A. Analysis of the relationship between PDHX expression and tumor-associated fibroblasts and immune infiltration .B. Different algorithms were used to explore the potential correlation between the expression level of PDHX gene and the invasion level of tumor-associated fibroblasts in all types of tumors in TCGA. In TCGA, there is a potential correlation between the expression level of PDHX gene and the infiltration level of tumor-associated fibroblasts.

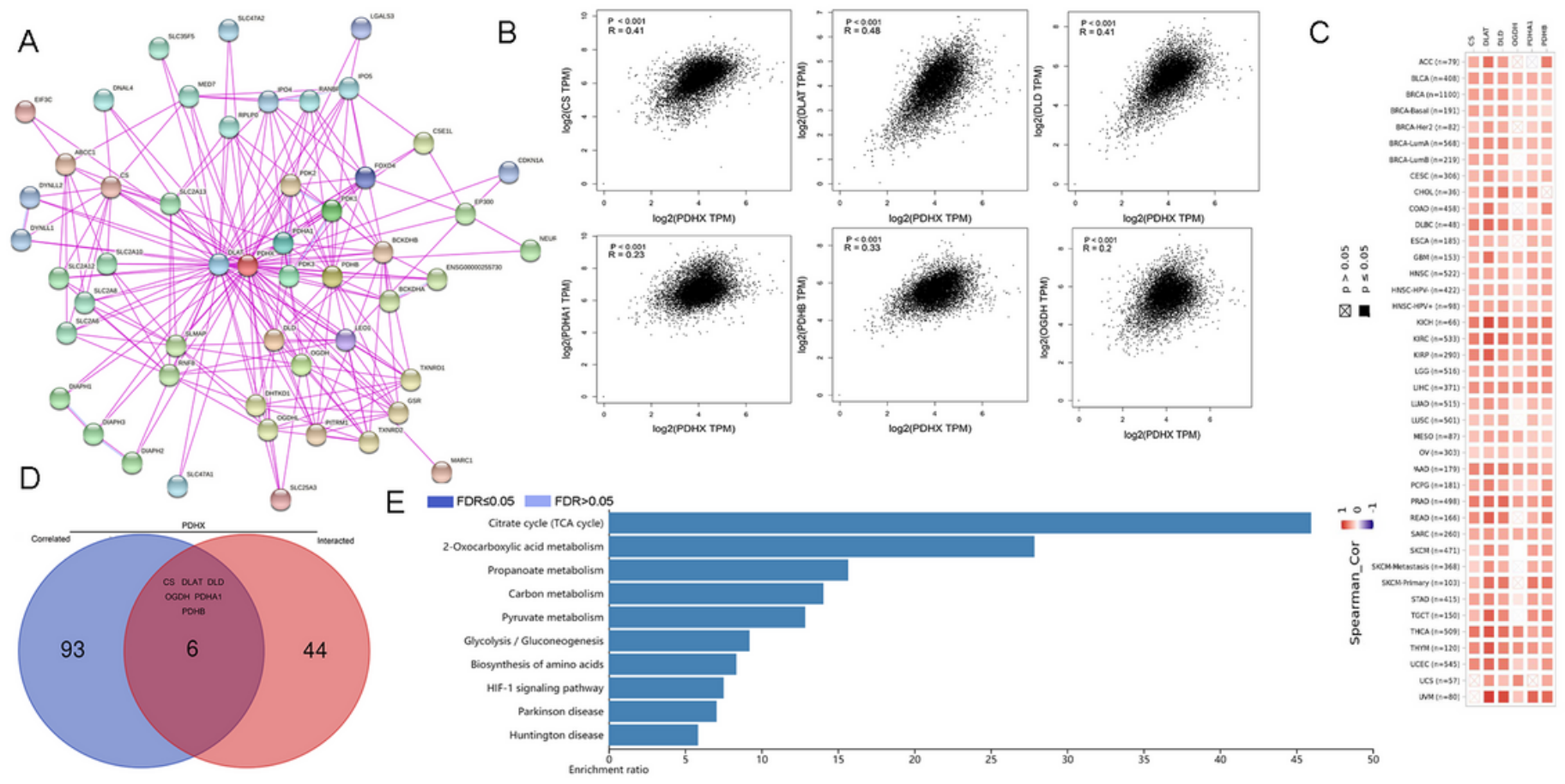


Figure 4

A. We first screened 50 available PDHX-binding proteins using the STRING tool. B. Using GEPIA2 database, we also obtained the first 100 genes related to PDHX in TCGA database, and analyzed the expression correlation between PDHX and selected target genes CS, DLAT, DLD, OGDH, PDHA1 and PDHB .C. Shows the heat map data of the previous related genes in the detailed cancer types. D. Cross analysis of PDHX binding genes and related genes.E. Based on the PDHX-binding and interacted genes, KEGG pathway analysis was performed.

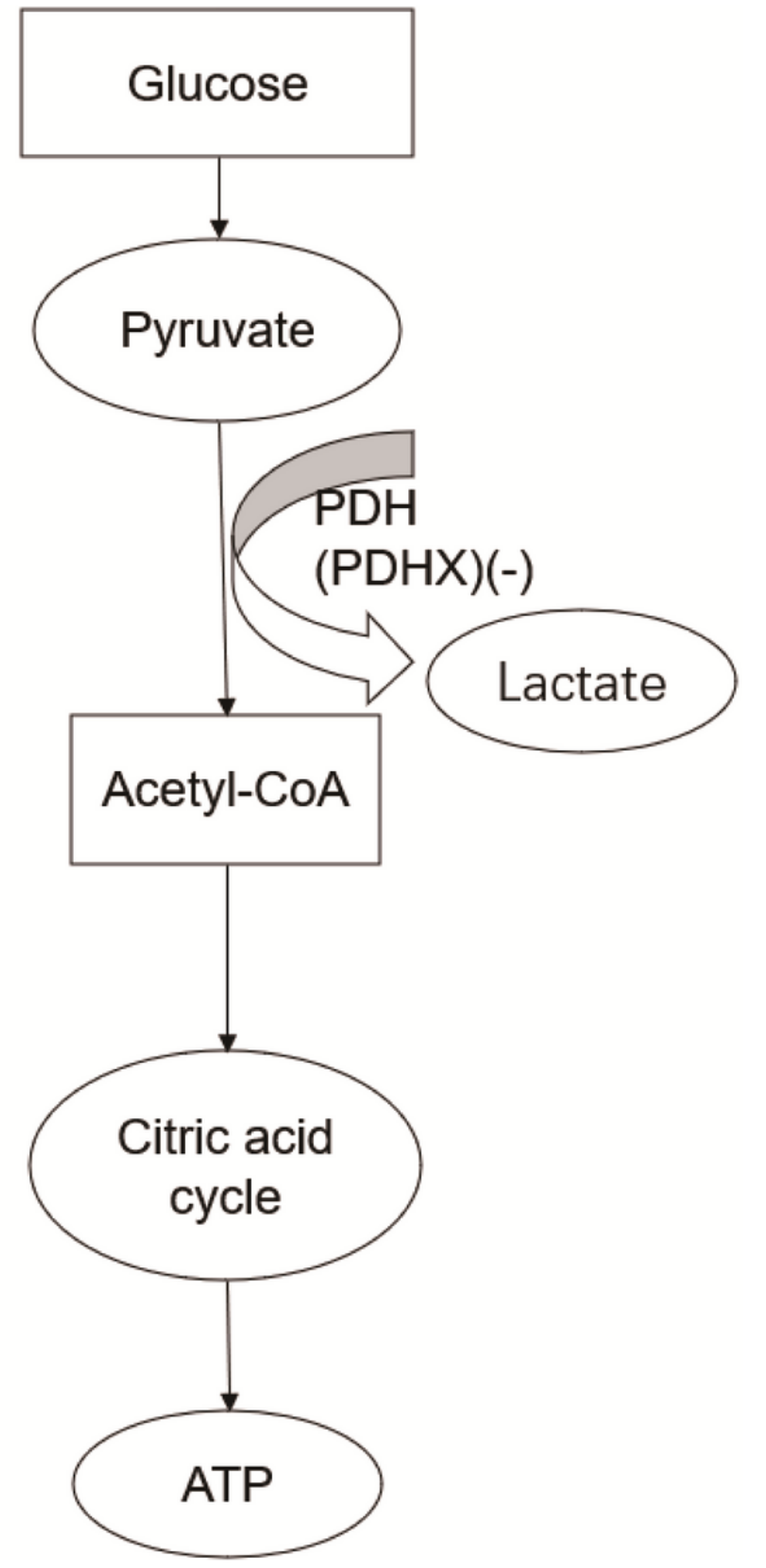


Figure 5

PDHX is involved in the molecular mechanism of glucose metabolism in gastric cancer cells. Low expression of PDHX inhibits the conversion of pyruvate to acetyl-CoA and makes pyruvate more converted to lactic acid, thus blocking the key step of glycolysis to tricarboxylic acid cycle. "-" stands for inhibitory effect. 